\title{
Inertial and bias effects in the rotational Brownian motion of rodlike molecules in a uniaxial potential
}

\author{
Yuri P. Kalmykov, ${ }^{1, a)}$ Sergey V. Titov, ${ }^{2}$ and William T. Coffey ${ }^{3}$ \\ ${ }^{1}$ LAMPS, Université de Perpignan Via Domitia, 52, Avenue Paul Alduy, 66860, Perpignan Cedex, France \\ ${ }^{2}$ Kotel'nikov Institute of Radio Engineering and Electronics of the Russian Academy of Sciences, Vvedenskii \\ Square 1, Fryazino, Moscow Region, 141190, Russian Federation \\ ${ }^{3}$ Department of Electronic and Electrical Engineering, Trinity College, Dublin 2, Ireland
}

(Received 11 October 2010; accepted 16 November 2010; published online 28 January 2011)

\begin{abstract}
Inertial effects in the rotational Brownian motion in space of a rigid dipolar rotator (needle) in a uniaxial potential biased by an external field giving rise to asymmetry are treated via the infinite hierarchy of differential-recurrence relations for the statistical moments (orientational correlation functions) obtained by averaging the Euler-Langevin equation over its realizations in phase space. The solutions of this infinite hierarchy for the dipole correlation function and its characteristic times are obtained using matrix continued fractions showing that the model simultaneously predicts both slow overbarrier (or interwell) relaxation at low frequencies accompanied by intermediate frequency Debye relaxation due to fast near-degenerate motion in the wells of the potential (intrawell relaxation) as well as the high frequency resonance (Poley) absorption due to librations of the dipole moments. It is further shown that the escape rate of a Brownian particle from a potential well as extended to the Kramers turnover problem via the depopulation factor yields a close approximation to the longest (overbarrier) relaxation time of the system. For zero and small values of the bias field parameter $h$, both the dipole moment correlation time and the longest relaxation time have Arrhenius behavior (exponential increase with increasing barrier height). While at values of $h$ in excess of a critical value however far less than that required to achieve nucleation, the Arrhenius behavior of the correlation time disappears. () 2011 American Institute of Physics. [doi:10.1063/1.3524534]
\end{abstract}

\section{INTRODUCTION}

The inertial rotational Brownian motion in space in an asymmetric double-well potential occurs in many physical problems involving short-time (high-frequency) orientational relaxation processes in liquids and solids, e.g., dielectric relaxation in molecular and nematic liquid crystals, dynamic Kerr effect in liquids, magnetic relaxation of ferrofluids (colloidal suspensions of single-domain ferromagnetic particles), etc. ${ }^{1-3}$ One of the most frequently used models for the interpretation of these phenomena is the Langevin or FokkerPlanck model for rotational diffusion of particles in fluids. This model relies on the solution of the appropriate EulerLangevin equation for the angular velocity or the corresponding Fokker-Planck equation for the angular momentumorientation conditional probability distribution function. In particular, the Fokker-Planck equation describes the time evolution of the distribution function of orientations and momenta in phase space while the Euler-Langevin equation is a stochastic vector differential equation for the angular velocity random variables describing their rotational dynamics. Both approaches are entirely equivalent so that all observables of interest can be calculated from either equation and in both cases can be reduced to the task of solving a hierarchy of differential-recurrence equations for the statistical averages describing the dynamical behavior of the appropriate physical

\footnotetext{
a) Author to whom correspondence should be addressed. Electronic mail: kalmykov@univ-perp.fr.
}

quantities. In order to determine these averages one can use various mathematical methods to solve the hierarchy notably continued fractions, yielding solutions in a tractable form. ${ }^{1,2}$

Now one of the most important characteristics associated with the Brownian motion in any asymmetric doublewell potential is the dependence of the longest relaxation time on the asymmetry or bias parameter which can be varied by altering the magnitude of an external field. The longest relaxation time is the time required to escape the well or the relaxation time of the longest lived relaxation mode. It is the inverse of the smallest nonvanishing eigenvalue $\lambda_{1}$ of the governing Fokker-Planck equation or equivalently the inverse of the smallest root of the secular equation of the hierarchy of differential-recurrence equations. One may also introduce the integral relaxation time which is the area under the appropriate relaxation (correlation) function following the removal of a steady field. Now it is known from the theory of the Brownian motion that in a symmetric doublewell potential, the integral relaxation time can be accurately approximated by the longest relaxation time. However, in a potential with nonequivalent wells the longest relaxation time, above a certain value of the bias field that is considerably less than that needed to achieve nucleation, may exponentially diverge from the integral relaxation time. ${ }^{2}$ This interesting effect arises due to depletion of the population of the shallower of the two wells by the prolonged action of the bias field. Thus the symmetric bistable potential masks remarkable effects of the exponential divergence of the integral relaxation time and the longest relaxation time that may appear for 
potentials with nonequivalent wells. There the asymmetry of the potential can radically alter the characteristics of the relaxation process. ${ }^{2}$ Hence it is of some importance to establish the effect of the asymmetry parameter on the relaxation behavior.

As far as the calculation of the longest relaxation time is concerned, the secular equation governing the eigenvalues of the decay modes of the system may be generated by averaging the appropriate Langevin equation over its realizations in phase space. This procedure yields a hierarchy of differentialrecurrence equations whence one may determine the secular equation. ${ }^{2}$ Alternatively, one may separate variables in the Fokker-Planck equation written in terms of the observables in order to derive the same hierarchy ${ }^{1}$ via the appropriate orthonormal basis. Thus $\lambda_{1}$ is not in general available in closed form as it is always rendered as the smallest root of an infinite order polynomial equation. Hence an approximate closed form expression for $\lambda_{1}$ is desirable. This may be accomplished by considering the thermally activated escape of particles out of a potential well giving rise to an ingenious method of estimating $\lambda_{1}$ originally proposed by Kramers. ${ }^{4}$ His approach relies on the fact that for a high potential barrier $\lambda_{1}$ has the same asymptotic behavior as the escape rate over the barrier. Thus Kramers evaluated in the high barrier limit the prefactor $A$ in an Arrhenius-like equation for the escape rate $\Gamma$ over a potential barrier $\Delta V$ (reaction velocity $\Gamma$ in the case of chemical reactions), viz.,

$$
\Gamma=A \frac{\omega_{a}}{2 \pi} e^{-\Delta V / k_{B} T},
$$

where the attempt frequency, $\omega_{a}$, is the angular frequency of a particle executing oscillatory motion at the bottom of a well (for reviews of applications of Kramers' method see, e.g., Refs. 5-9). Now Kramers ${ }^{4}$ originally obtained so called intermediate-to-high damping (IHD) and very low damping (VLD) formulas for the escape rate, assuming in both cases that the energy barrier is much greater than the thermal energy so that the concept of an escape rate applies. We remark that the limiting cases of IHD are intermediate damping (ID), where the escape rate is independent of the damping, corresponding to the results of classical transition state theory (TST) and very high damping (VHD), where the escape rate is inversely proportional to the damping. Kramers could not, however, find a formula describing the escape rate in the region (now dubbed the Kramers turnover region) between ID and VLD damping. Much later an escape rate formula valid for any damping regime was proposed by Mel'nikov and Meshkov. ${ }^{10-12}$ This involves a depopulation factor that interpolates for the escape rate between the VLD and ID regimes. They then postulated that a formula valid in all damping regimes could be written by simply taking the product of the depopulation factor and the IHD escape rate. Subsequently, Grabert $^{13}$ and Pollak et al. ${ }^{14}$ presented a complete solution of the Kramers turnover problem showing that their formula can be obtained without ad hoc interpolation between the weak and ID damping regimes. The turnover formula very favorably compares with calculations based on either the solution of the Fokker-Planck equation or on numerical simulations of the Brownian dynamics for various stochastic systems (the escape out of a single well, ${ }^{15,16}$ translational Brownian mo- tion in a potential, ${ }^{17-19}$ the diffusion on a surface, ${ }^{20-22}$ the rotational Brownian motion in double-well potentials ${ }^{23-26}$ ). Furthermore, Coffey et al. ${ }^{9,27}$ (using escape rate theory) and Denisov et al. ${ }^{28}$ (applying the mean first passage time approach) extended the calculation to the reversal time of the magnetization in superparamagnets (classical giant spins). We remark that the magnetization relaxation problem differs fundamentally from that of mechanical particles because the undamped equation of motion of the magnetization of a single domain ferromagnetic particle is the gyromagnetic equation and the Hamiltonian is no longer separable and additive. Thus the inertia plays no role; the part played by inertia in the mechanical system is essentially mimicked in the magnetic system for nonaxially symmetric potentials by the gyromagnetic term which gives rise to the coupling or entanglement of the transverse and longitudinal modes leading ${ }^{2}$ to ferromagnetic resonant behavior in the transverse relaxation and to the various Kramers damping regions in the longitudinal relaxation.

Here we shall consider, as a particular example, the inertial rotational Brownian motion of rodlike molecules (rigid rotators) in space in an axially symmetric potential with nonequivalent wells, viz.,

$$
V(\vartheta)=-\sigma k_{B} T\left(\cos ^{2} \vartheta+2 h \cos \vartheta\right),
$$

where $\sigma$ is the dimensionless anisotropy or barrier height parameter, $h=\xi /(2 \sigma)$ is the dimensionless external field (asymmetry) parameter, $\xi=\mu E /\left(k_{B} T\right), k_{B}$ is Boltzmann's constant, $T$ is the temperature, and $\vartheta$ is the polar angle (colatitude). In the present context of relaxation in asymmetrical potential wells we remark that most results have been obtained for potentials with equivalent wells. For example, the rotational Brownian motion of rodlike molecules in space in a symmetric uniaxial (Meier-Saupe) potential $V(\vartheta)=-\sigma k_{B} T \cos ^{2} \vartheta$ is almost invariably used to describe the molecular reorientations in nematic liquid crystals (see, e.g., Refs. 29-34) and biological membranes (e.g., Refs. 35 and 36). Moreover, nearly all treatments were confined to the noninertial or VHD limit so that the Smoluchowski equation in configuration space could be used (see, e.g., Refs. 30-33 and references cited therein). In the orientational relaxation in molecular liquids, the inertial effects of the molecules are unimportant at low frequencies, $\omega \tau<1$, where $\tau$ is the orientational relaxation time. However, neglecting inertia leads to incorrect predictions at high frequencies (e.g., infinite integral absorption in dielectric spectra). ${ }^{2}$ Finally, in view of the difficulties in incorporating a potential combined with rotation in space, treatments including inertial effects have been usually limited either to numerical simulation ${ }^{3,23,37}$ or rotation about a fixed axis. $24,25,38$

Now as we saw the theoretical treatment of inertial rotational Brownian motion has been usually based on the Fokker-Planck equation (which for separable and additive Hamiltonians is called the Klein-Kramers equation) for the evolution of the distribution function in configuration-angular velocity space. ${ }^{39,40}$ An alternative approach to the problem has been given by Coffey ${ }^{41}$ and Coffey et al. ${ }^{2}$ They developed a method of solution for the observables (relaxation functions, etc.) via the Euler-Langevin equation for the inertial 
Brownian rotation which does not involve the Fokker-Planck equation. The key step ${ }^{41}$ in applying the method is first to convert the Langevin equation into an equation for the quantity the statistical average of which is desired. The Langevin equation in the new variables is then averaged over its realizations in phase space yielding the observables. This procedure entirely eliminates the excessive step in the theory of constructing and solving the corresponding Fokker-Planck equation for the probability density function. The advantages of the Langevin equation method for the rotational Brownian motion in space in the presence of a potential have been amply demonstrated in Refs. 26 and 42, where inertial effects in the orientational relaxation have been treated.

Here we apply this averaging method to the evaluation of the inertial response of systems of polar molecules in space in an asymmetrical double-well potential, Eq. (2), with dynamics described by the inertial Euler-Langevin equation. We shall first show how the solution of the corresponding recurrence relations for the statistical moments can be obtained using matrix continued fractions yielding, in particular, the exact solution for the dynamic longitudinal susceptibility. Furthermore, we shall present approximate solutions by utilizing the concepts of the integral relaxation (correlation) time and escape rate. ${ }^{2}$ We shall also present a detailed comparison of the results for the longest relaxation time yielded by the turnover formula with the exact matrix continued fraction solution for the first rank orientational correlation function, its correlation time, and generalized dynamic susceptibility. We shall also demonstrate that the turnover formula ${ }^{10,11}$ when applied to the particular problem of the inertial Brownian motion of a rigid rotator in an asymmetric double-well potential yields an accurate solution for the longest relaxation time for high barriers and for all values of the dissipation. Such a potential takes account of flipping of rotators to neighboring wells, thus permitting both overbarrier (i.e., interwell) and intrawell relaxation as well as resonance absorption due to fast oscillations in the wells of the potential. Thus the model may simultaneously explain both the low-frequency relaxation and the far-infrared absorption spectra of dipolar systems. Finally, we shall show that introducing asymmetry will cause the overbarrier mode to become so weak at a certain critical value of the asymmetry parameter that it will be almost completely suppressed while both intrawell relaxation and oscillatory modes remain.

\section{BASIC RELATIONS}

We consider the three-dimensional rotational diffusion of a thin rod, or rigid rotator, representing the linear polar molecule, in space in a potential Eq. (2). In the molecular coordinate system oxyz fixed in the rotator, the rotational Brownian motion of the rotator is governed by the vector Euler-Langevin equation in moving axes ${ }^{40,43}$

$$
\frac{d}{d t} \mathbf{L}(t)+\omega(t) \times \mathbf{L}(t)+\zeta \omega(t)=-\nabla V+\lambda(t),
$$

where $\boldsymbol{\omega}$ and $\mathbf{L}$ are the angular velocity and the angular momentum of the rotator, respectively, $-\nabla V$ is the torque due to the external potential $V, \nabla \equiv \delta / \delta \varphi$ is the orientation space gradient operator and $\delta \varphi$ is an infinitesimal rotation vector so that $\boldsymbol{\omega}(t)=\delta \boldsymbol{\varphi} / \delta t, \zeta \omega(t)$ and $\lambda(t)$ are the frictional and white noise torques due to the Brownian motion of the surroundings, and $\zeta$ is the rotational friction tensor. The angular velocity $\boldsymbol{\omega}=\left(\omega_{x}, \omega_{y}, \omega_{z}\right)$ and the angular momentum $\mathbf{L}=\left(I \omega_{x}, I \omega_{y}, 0\right)$ of the rotator may be defined as ${ }^{40}$

$$
\boldsymbol{\omega}=(\dot{\vartheta}, \dot{\varphi} \sin \vartheta, \dot{\varphi} \cos \vartheta) \quad \text { and } \quad \mathbf{L}=(I \dot{\vartheta}, I \dot{\varphi} \sin \vartheta, 0) \text {, }
$$

where $\varphi$ is the azimuthal angle and $I$ is the moment of inertia of the rotator about the axis of symmetry. Here the component $\omega_{z}$ of the angular velocity $\omega$ is not an independent variable; it is determined by the equation $\omega_{z}^{2}=\omega^{2}-\omega_{x}^{2}-\omega_{y}^{2}$, where $\boldsymbol{\omega}^{2}=\dot{\vartheta}^{2}+\dot{\varphi}^{2}$. Now Eq. (3) rewritten in terms of the angular velocity components $\omega_{x}$ and $\omega_{y}$ becomes

$I \dot{\omega}_{x}(t)=I \omega_{y}^{2}(t) \cot \vartheta(t)-\zeta \omega_{x}(t)-\partial_{\vartheta} V[\vartheta(t)]+\lambda_{x}(t)$,

$I \dot{\omega}_{y}(t)=-I \omega_{x}(t) \omega_{y}(t) \cot \vartheta(t)-\zeta \omega_{y}(t)+\lambda_{y}(t)$,

where $-\partial_{\vartheta} V[\vartheta(t)]$ is the conservative torque due to the external potential $V, \zeta$ is the friction coefficient for rotation about the $x$ and $y$ axes. The random torque components $\lambda_{x}(t)$ and $\lambda_{y}(t)$ have the following properties

$$
\overline{\lambda_{j}(t)}=0, \overline{\lambda_{j}(t) \lambda_{m}\left(t^{\prime}\right)}=2 k_{B} T \zeta \delta_{j, m} \delta\left(t-t^{\prime}\right),
$$

where $j, m=x, y$ and the overbar means the statistical average over an ensemble of rotators that all start at the same instant $t$ with the same sharp values of the angular velocity and the orientation.

Our goal is to evaluate the longitudinal dipole correlation function $C(t)$ defined as

$$
C(t)=\frac{\langle\cos \vartheta(0) \cos \vartheta(t)\rangle-\langle\cos \vartheta(0)\rangle^{2}}{\left\langle\cos ^{2} \vartheta(0)\right\rangle-\langle\cos \vartheta(0)\rangle^{2}},
$$

where the angular brackets mean the equilibrium statistical average. Having determined $C(t)$ from Eq. (6), one may calculate the correlation (or integral relaxation) time $\tau$ defined as the area under the curve of the correlation function, viz.,

$$
\tau=\int_{0}^{\infty} C(t) \mathrm{d} t
$$

and the longitudinal complex susceptibility $\chi(\omega)=\chi^{\prime}(\omega)-$ $i \chi^{\prime \prime}(\omega)$ defined as

$$
\chi(\omega) / \chi^{\prime}(0)=1-i \omega \int_{0}^{\infty} e^{-i \omega t} C(t) \mathrm{d} t,
$$

where

$$
\chi^{\prime}(0)=\frac{\mu^{2} N_{0}}{k_{B} T}\left[\left\langle\cos ^{2} \vartheta(0)\right\rangle-\langle\cos \vartheta(0)\rangle^{2}\right]
$$

is the static susceptibility and $N_{0}$ is the number of dipoles per unit volume.

The calculation of the correlation function $C(t)$ can be accomplished by solving the infinite hierarchy of differentialrecurrence relations for the correlation functions

$$
c_{n}^{l, m}(t)=\left\langle\cos \vartheta(0) f_{n}^{l, m}(t)\right\rangle-\langle\cos \vartheta(0)\rangle\left\langle f_{n}^{l, m}(0)\right\rangle ;
$$


this hierarchy has been derived for an arbitrary axially symmetric potential $V(\vartheta)$ in Ref. 42 by averaging the Langevin Eqs. (4) and (5). Here

$$
f_{n}^{l, m}(t)=P_{l}^{m}[\cos \vartheta(t)] s_{n}^{m}\left[\omega_{x}(t), \omega_{y}(t)\right],
$$

where $P_{l}^{k}(z)$ are the associated Legendre functions ${ }^{44}$ and the orthogonal functions $s_{n}^{m}\left(\omega_{x}, \omega_{y}\right)$ may be expressed as a finite series of products of Hermite polynomials $H_{n}(z)$ in the components of the angular velocity, viz., ${ }^{45}$

$$
\begin{aligned}
s_{n}^{2 m+M} & \left(\omega_{x}, \omega_{y}\right) \\
& =\sum_{q=0}^{n} \frac{r_{2 m+M}(n, q)}{q !(n-q) !} H_{2 n-2 q+M}\left(\eta \omega_{x}\right) H_{2 q}\left(\eta \omega_{y}\right),
\end{aligned}
$$

where $M=0$ or 1 . Here the coefficients $r_{2 m+M}(n, q)$ are to be determined from the following recurrence relations

$$
\begin{aligned}
r_{2 m}(n, q)= & 2\left(n-q+\frac{1}{2}\right)\left(\frac{m-q-1}{2 m-1}\right) r_{2 m-1}(n, q) \\
& +(n-q) \frac{2 q+1}{2 m-1} r_{2 m-1}(n, q+1)
\end{aligned}
$$

$$
r_{2 m+1}(n, q)=\frac{q+m}{m} r_{2 m}(n, q)-\frac{q}{m} r_{2 m}(n, q-1)
$$

with $r_{0}(n, q)=r_{1}(n, q)=1$. Similar functions are also implicit in the work of $\mathrm{Sack}^{46}$ who used a representation of the angular velocity distribution function in terms of characteristic functions. For the potential of Eq. (2), the general differential-recurrence equations derived in Ref. 42 become for $m=0$

$$
\begin{aligned}
\eta \frac{d}{d t} c_{n}^{l, 0}= & -2 n \beta^{\prime} c_{n}^{l, 0}+\frac{1}{2} c_{n}^{l, 1}+\left(2+\frac{4 \sigma}{(2 l-1)(2 l+3)}\right) c_{n-1}^{l, 1} \\
& -\frac{2 \xi}{2 l+1}\left(c_{n-1}^{l-1,1}-c_{n-1}^{l+1,1}\right) \\
& -\frac{4 \sigma}{2 l+1}\left(\frac{l}{2 l-1} c_{n-1}^{l-2,1}-\frac{l+1}{2 l+3} c_{n-1}^{l+2,1}\right)
\end{aligned}
$$

and for $m \geq 1, M=0,1$

$$
\begin{aligned}
\eta \frac{d}{d t} c_{n}^{l, 2 m-M}= & -(2 n+M) \beta^{\prime} c_{n}^{l, 2 m-M}+c_{n-1+M}^{l, 2 m+1-M}+\frac{1}{4} c_{n+M}^{l, 2 m+1-M}-(l+2 m-M)(l-2 m+1+M) \\
& \times\left[\frac{n-m+1+M}{4} c_{n+M}^{l, 2 m-1-M}+(n+m) c_{n-1+M}^{l, 2 m-1-M}\right]-\frac{\xi}{2 l+1}\left\{c_{n-1+M}^{l-1,2 m+1-M}-c_{n-1+M}^{l+1,2 m+1-M}\right. \\
& \left.-(n+m)\left[(l+2 m-1-M)(l+2 m-M) c_{n-1+M}^{l-1,2 m-1-M}-(l-2 m+1+M)(l-2 m+2+M) c_{n-1+M}^{l+1,2 m-1-M}\right]\right\} \\
& +2 \sigma\left\{\frac{(l+1-2 m+M)}{(2 l+1)(2 l+3)} c_{n-1+M}^{l+2,2 m+1-M}+\frac{(4 m-2 M+1)}{(2 l-1)(2 l+3)} c_{n-1+M}^{l, 2 m+1-M}-\frac{(l+2 m-M)}{(2 l-1)(2 l+1)} c_{n-1+M}^{l-2,2 m+1-M}\right. \\
& -(n+m)\left[\frac{(l-2 m+1+M)(l-2 m+2+M)(l-2 m+3+M)}{(2 l+1)(2 l+3)} c_{n-1+M}^{l+2,2 m-1-M}\right. \\
& \left.-\frac{(4 m-2 M-1)(l-2 m+1+M)(l+2 m-M)}{(2 l-1)(2 l+3)} c_{n-1+M}^{l, 2 m-1-M}\right] \\
& \left.\left.-\frac{(l+2 m-2-M)(l+2 m-1-M)(l+2 m-M)}{(2 l-1)(2 l+1)} c_{n-1+M}^{l-2,2 m-1-M}\right]\right\}
\end{aligned}
$$

where $\eta=\sqrt{I /\left(2 k_{B} T\right)}$ and $\beta^{\prime}=\zeta \eta / I$ is the normalized friction.

Equations (13) and (14) may now be solved for the onesided Fourier transform $\tilde{c}_{0}^{1,0}(i \omega)=\int_{0}^{\infty} c_{0}^{1,0}(t) e^{-i \omega t} d t$ using matrix continued fractions as described in the Appendix. Having determined $\tilde{c}_{0}^{1,0}(i \omega)$, we can calculate the spectrum $\tilde{C}(\omega)$ of the autocorrelation function $C(t)$ and the correlation (integral relaxation) time $\tau=\tilde{C}(0)$, viz.,

$$
\tilde{C}(\omega)=\frac{\tilde{c}_{0}^{1,0}(i \omega)}{c_{0}^{1,0}(0)} \quad \text { and } \quad \tau=\frac{\tilde{c}_{0}^{1,0}(0)}{c_{0}^{1,0}(0)}
$$

We may also approximately evaluate the longest relaxation time $\tau_{L}=1 / \lambda_{1}$ by using $\tilde{C}(\omega)$ for moderate asymmetry as follows. First consider the long time behavior of $C(t)$ corresponding to the low frequency behavior of $\tilde{C}(\omega)$. The autocorrelation function $C(t)$ can be approximated at long times by an exponential ${ }^{18}$

$$
C(t) \approx C_{0} e^{-t / \tau_{L}} \text {. }
$$

Thus the characteristic (longest) relaxation time $\tau_{L}$ for moderate asymmetry can then be extracted from $\tilde{C}(\omega)$ (by eliminating $C_{0}$ ) as

$$
\tau_{L}=\lim _{\omega \rightarrow 0} \frac{\tilde{C}(0)-\tilde{C}(\omega)}{i \omega \tilde{C}(\omega)} .
$$


Equations (16) and (17) imply by transposition that the lowfrequency part of the spectrum $\tilde{C}(\omega)$ may be approximated in the context of a rate description by the single Lorentzian

$$
\tilde{C}(\omega) \approx \frac{\tilde{C}(0)}{1+i \omega \tau_{L}} .
$$

On the other hand by utilizing general properties of Fourier transforms, we can also obtain the asymptotic expansion for $\tilde{C}(\omega)$ in the high-frequency limit, $\omega \rightarrow \infty$, viz.,

$$
\tilde{C}(\omega)=\int_{0}^{\infty} C(t) e^{-i \omega t} \mathrm{~d} t \sim-\frac{i}{\omega}-\frac{i \ddot{C}(0)}{\omega^{3}}+\frac{\dddot{C}(0)}{\omega^{4}} \cdots
$$

so that

$$
\operatorname{Im}[\tilde{C}(\omega)] \sim-\omega^{-1}+\cdots \text { and } \operatorname{Re}[\tilde{C}(\omega)] \sim \dddot{C}(0) \omega^{-4}+\cdots
$$

(here we have noted that $C(0)=1$ and $\dot{C}(0)=\dot{c}_{0}^{1,0}(0)$ / $\left.c_{0}^{1,0}(0)=0\right)$. Hence we have the limiting behavior at low and high frequencies.

\section{ASYMPTOTIC FORMULAS FOR THE LONGEST RELAXATION TIME}

The longest relaxation time can also be analytically evaluated using escape rate theory as $\Gamma^{-1}$, where $\Gamma$ is the sum of the forward and backward escape rates (insofar as a rate description ${ }^{10,47}$ applies). Here the escape rate turnover formula can be obtained by generalizing the Pastor and $\mathrm{Szabo}^{23}$ method of calculation of $\Gamma$ for a uniaxial potential with equivalent wells. They accomplished this by averaging over one of the angular velocity components $\omega_{y}$ in the exact inertial Fokker-Planck equation (rotational Klein-Kramers equation) for the distribution function $W\left(\vartheta, \omega_{x}, \omega_{y}, t\right)$, which is

$$
\frac{\partial W}{\partial t}=L_{\mathrm{FP}} W
$$

where the Fokker-Planck operator $L_{\mathrm{FP}}$ is defined as

$$
\begin{aligned}
L_{\mathrm{FP}}= & -\omega_{x} \partial_{\vartheta}-\omega_{y} \cot \vartheta\left(\omega_{y} \partial_{\omega_{x}}-\omega_{x} \partial_{\omega_{y}}\right)+I^{-1} \partial_{\vartheta} V \partial_{\omega_{x}} \\
& +\frac{\beta^{\prime}}{\eta}\left[\partial_{\omega_{x}}\left(\omega_{x}+\frac{1}{2 \eta^{2}} \partial_{\omega_{x}}\right)+\partial_{\omega_{y}}\left(\omega_{y}+\frac{1}{2 \eta^{2}} \partial_{\omega_{y}}\right)\right] .
\end{aligned}
$$

The averaging procedure allowed them to reduce Eq. (21) to a simpler Fokker-Planck equation for a reduced distribution function $\quad \bar{W}\left(\vartheta, \omega_{x}, t\right)=\sin \vartheta \frac{\eta}{\sqrt{\pi}} \int_{-\infty}^{\infty} e^{-\eta^{2} \omega_{y}^{2}}$ $W\left(\vartheta, \omega_{x}, \omega_{y}, t\right) d \omega_{y}$, viz.,

$\frac{\partial \bar{W}}{\partial t}+\dot{\vartheta} \frac{\partial \bar{W}}{\partial \vartheta}-\frac{1}{I} \frac{\partial \bar{V}}{\partial \vartheta} \frac{\partial \bar{W}}{\partial \dot{\vartheta}}=\frac{\beta^{\prime}}{\eta} \frac{\partial}{\partial \dot{\vartheta}}\left(\dot{\vartheta} \bar{W}+\frac{1}{2 \eta^{2}} \frac{\partial \bar{W}}{\partial \dot{\vartheta}}\right)$,

where $\bar{V}(\vartheta)=V(\vartheta)-k_{B} T \ln \sin \vartheta$ is the effective potential (this potential is shown in Fig. 1). Here the potential $\bar{V}(\vartheta)$ is infinite at 0 and $\pi$, has a maximum at $\vartheta=\vartheta_{\max }$ in that interval, and so resembles a typical one-dimensional asymmetric bistable potential in the range $0<\vartheta<\pi$. Clearly the Fokker-Planck Eq. (23) describes the one-dimensional Brownian rotation about a fixed axis in the effective potential $\bar{V}(\vartheta)$

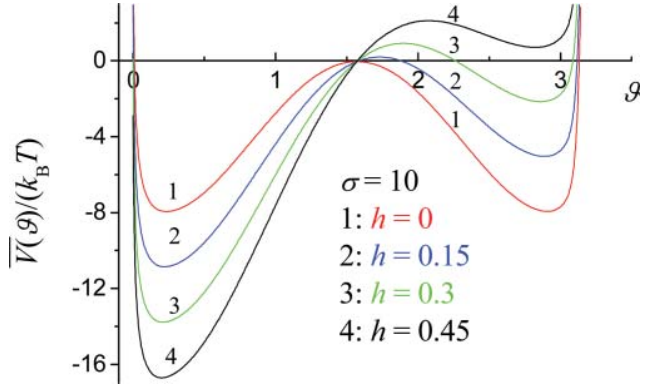

FIG. 1. The effective potential $\bar{V}(\vartheta) / k_{B} T=-\sigma \cos ^{2} \vartheta-\xi \cos \vartheta-$ $\ln \sin \vartheta$.

and is similar to the Klein-Kramers equation describing the translational Brownian motion of a point particle. Hence, the translational depopulation factor is directly applicable without modification ${ }^{24,25}$ and so one obtains the longest relaxation time $\tau_{M}=\Gamma^{-1}$ (which may be used to estimate $\tau_{L}$ ) for all values of the damping as

$$
\tau_{M}=\frac{A\left[\beta^{\prime}\left(S_{1}+S_{2}\right)\right] \eta \omega_{b}\left(\Gamma_{1}^{\mathrm{TST}}+\Gamma_{2}^{\mathrm{TST}}\right)^{-1}}{A\left(\beta^{\prime} S_{1}\right) A\left(\beta^{\prime} S_{2}\right)\left(\sqrt{\beta^{\prime 2}+4 \eta^{2} \omega_{b}^{2}}-\beta^{\prime}\right)},
$$

where $\omega_{b}=\sqrt{\left|\partial_{\vartheta}^{2} \bar{V}\left[\vartheta_{\max }\right]\right| / I} \sim \eta^{-1} \sqrt{\sigma\left(1-h^{2}\right)}$ is the barrier frequency and the TST escape rates $\Gamma_{1}^{\mathrm{TST}}$ and $\Gamma_{2}^{\mathrm{TST}}$ from each of the two wells are

$$
\begin{aligned}
\Gamma_{1}^{\mathrm{TST}} & \sim \frac{e^{-\bar{V}\left[\vartheta_{\max }\right] / k_{B} T}}{4 \eta \sqrt{\pi} \int_{0}^{\vartheta_{\max }} e^{-\bar{V}(\vartheta) / k_{B} T} \mathrm{~d} \vartheta} \\
& \sim \frac{\sigma(1+h)}{2 \eta \sqrt{\pi}} \sqrt{1-h^{2}} e^{-\sigma(1+h)^{2}}, \\
\Gamma_{2}^{\mathrm{TST}} & \sim \frac{e^{-\bar{V}\left[\vartheta_{\max }\right] / k_{B} T}}{4 \eta \sqrt{\pi} \int_{\vartheta_{\max }}^{\pi} e^{-\bar{V}(\vartheta) / k_{B} T} \mathrm{~d} \vartheta} \\
& \sim \frac{\sigma(1-h)}{2 \eta \sqrt{\pi}} \sqrt{1-h^{2}} e^{-\sigma(1-h)^{2}} .
\end{aligned}
$$

Here the subscripts 1 and 2 correspond to the shallow and deep well, respectively, $\vartheta_{\max }$ is the root of the equation $\partial_{\vartheta} \bar{V}(\vartheta)=0$ corresponding to the maximum of the potential so that the domain of each well is specified by $\bar{V}\left(\vartheta_{\max }\right)-\bar{V}(\vartheta) \geq 0$. The maximum may be estimated as $\vartheta_{\max } \approx \arccos (-h)$, the $S_{1}$ and $S_{2}$ are the dimensionless action variables ${ }^{12,23}$ defined in our notation as

$$
\begin{aligned}
& S_{1}=4 \int_{\vartheta_{1}}^{\vartheta_{\max }} \sqrt{\left[\bar{V}\left(\vartheta_{\max }\right)-\bar{V}(\vartheta)\right] /\left(k_{B} T\right)} \mathrm{d} \vartheta \\
& S_{2}=4 \int_{\vartheta_{\max }}^{\vartheta_{2}} \sqrt{\left[\bar{V}\left(\vartheta_{\max }\right)-\bar{V}(\vartheta)\right] /\left(k_{B} T\right)} \mathrm{d} \vartheta .
\end{aligned}
$$

Clearly $\vartheta_{1}$ and $\vartheta_{2}$ specifying the left-hand boundary of well 1 and the right-hand boundary of well 2 are the inferior and superior roots of the equation of the separatrix $\bar{V}\left(\vartheta_{\max }\right)=\bar{V}(\vartheta)$. Moreover,

$$
A(\delta)=\exp \left\{\frac{2}{\pi} \int_{0}^{\pi / 2} \ln \left[1-e^{-\delta /\left(4 \cos ^{2} x\right)}\right] \mathrm{d} x\right\}
$$


is the (Mel'nikov) depopulation factor involving the ratio $\delta$ of the energy of the almost periodic motion of an escaping particle to the thermal energy. The actions $S_{1}$ and $S_{2}$ are calculated on the critical energy contour or separatrix on which a typical rotator librating ${ }^{48}$ in a well with energy equal to the barrier energy $\bar{V}\left(\vartheta_{\max }\right)$ may either by dint of thermal fluctuations escape from the well or return to its depths. On simplification of Eqs. (24)-(26), we have

$$
\tau_{M}=\frac{2 \sqrt{\pi / \sigma} A\left[\beta^{\prime}\left(S_{1}+S_{2}\right)\right]}{A\left(\beta^{\prime} S_{1}\right) A\left(\beta^{\prime} S_{2}\right)\left[(1+h) e^{-\sigma(1+h)^{2}}+(1-h) e^{-\sigma(1-h)^{2}}\right]\left[\sqrt{\beta^{\prime 2}+4 \sigma\left(1-h^{2}\right)}-\beta^{\prime}\right]} .
$$

The depopulation factor from Eq. (29) has the following properties:

$A\left(\beta^{\prime} S\right) \rightarrow 1$ as $\beta^{\prime} S \rightarrow \infty$ and $A\left(\beta^{\prime} S\right) / \beta^{\prime} S \rightarrow 1$ as $\beta^{\prime} S \rightarrow 0$.

Hence, it follows from these properties of $A\left(\beta^{\prime} S\right)$ that, in the low damping $\left(\beta^{\prime} \rightarrow 0\right)$ and very high damping $\left(\beta^{\prime} \rightarrow \infty\right)$ limits, Eq. (30) predicts

$\tau_{\mathrm{VLD}} \sim \frac{\left(S_{1}+S_{2}\right)\left[\left(1-h^{2}\right) / \pi\right]^{-1 / 2}}{\sigma \beta^{\prime} S_{1} S_{2}\left[(1+h) e^{-\sigma(1+h)^{2}}+(1-h) e^{-\sigma(1-h)^{2}}\right]}$

and

$\tau_{\mathrm{VHD}} \sim \eta \beta^{\prime} \frac{\sigma^{-3 / 2} \pi^{1 / 2}}{1-h^{2}}\left[(1-h) e^{-\sigma(1-h)^{2}}+(1+h) e^{-\sigma(1+h)^{2}}\right]^{-1}$.

For $h=0$, Eqs. (24)-(32) reduce to those for a uniaxial potential with two equivalent wells derived in Ref. 26.

We remark that in the low temperature limit, $\sigma \gg 1$, Eq. (32) coincides with the estimate of the longest relaxation time obtained ${ }^{2}$ from the axially symmetric Smoluchowski equation for the probability density function $W(\vartheta, t)$ of the orientations of rotators in space in a uniaxial potential, viz.,

$2 \tau_{D} \frac{\partial}{\partial t} W=\frac{1}{\sin \vartheta} \frac{\partial}{\partial \vartheta}\left[\sin \vartheta\left(\frac{\partial}{\partial \vartheta} W+\frac{W}{k_{B} T} \frac{\partial V}{\partial \vartheta}\right)\right]$,

where $\tau_{D}=\eta \beta^{\prime}$ is the Debye relaxation time for isotropic noninertial rotational diffusion. The Smoluchowski Eq. (33) in the angular variable $\vartheta$, originally due to Debye ${ }^{49}$ follows directly from the Fokker-Planck Eq. (21) by integrating over the angular momenta and proceeding to the VHD limit. In this limit, the correlation time can be given in closed form ${ }^{2}$

$$
\begin{aligned}
\tau= & \frac{2 \eta \beta^{\prime}}{Z\left(\left\langle\cos ^{2} \vartheta\right\rangle_{0}-\langle\cos \vartheta\rangle_{0}^{2}\right)} \\
& \times \int_{-1}^{1}\left[\int_{-1}^{z}\left(z^{\prime}-\langle\cos \vartheta\rangle_{0}\right) e^{\sigma\left(z^{\prime 2}+2 h z^{\prime}\right)} \mathrm{d} z^{\prime}\right]^{2} \frac{e^{-\sigma\left(z^{2}+2 h z\right)}}{1-z^{2}} \mathrm{~d} z
\end{aligned}
$$

where

$$
\langle\cos \vartheta\rangle_{0}=\frac{1}{Z} \int_{-1}^{1} z e^{\sigma\left(z^{2}+2 h z\right)} \mathrm{d} z=\frac{e^{\sigma} \sinh (2 \sigma h)}{\sigma Z}-h,
$$

$$
\begin{aligned}
\left\langle\cos ^{2} \vartheta\right\rangle_{0} & =\frac{1}{Z} \int_{-1}^{1} z^{2} e^{\sigma\left(z^{2}+2 h z\right)} \mathrm{d} z \\
Z & =\int_{-1}^{1} e^{\sigma\left(z^{2}+2 h z\right)} \mathrm{d} z \quad \sigma Z \\
& =\frac{e^{\sigma}[\cosh (2 \sigma h)-h \sinh (2 \sigma h)]}{2} \sqrt{\frac{\pi}{\sigma}} e^{-\sigma h^{2}}\{\operatorname{erfi}[(1+h) \sqrt{\sigma}]+\operatorname{erfi}[(1-h) \sqrt{\sigma}]\},
\end{aligned}
$$

and $\operatorname{erfi}(z)=(2 / \sqrt{\pi}) \int_{0}^{z} e^{t^{2}} d t$ is the error function of imaginary argument. A somewhat similar expression for the longest relaxation time $\tau_{L}$ in the VHD limit can be given via the mean first passage time ${ }^{6}$ (MFPT) as $\tau_{L}^{-1}=\left(\tau_{+}^{-1}+\tau_{-}^{-1}\right) / 2$, where $\tau_{+}$and $\tau_{-}$are the MFPTs for transitions from the minima of the potential Eq. (2) at $\vartheta=0$ and $\vartheta=\pi$ to the barrier point at $\vartheta=\arccos (-h)$, respectively. Hence ${ }^{2,6}$

$$
\begin{aligned}
\tau_{L}^{-1}= & \frac{1}{4 \tau_{D}}\left[\left(\int_{-1}^{-h} \frac{e^{-\sigma\left(z^{2}+2 h z\right)}}{1-z^{2}} \int_{-1}^{z} e^{\sigma\left(z^{\prime 2}+2 h z^{\prime}\right)} \mathrm{d} z^{\prime} \mathrm{d} z\right)^{-1}\right. \\
& \left.+\left(\int_{-h}^{1} \frac{e^{-\sigma\left(z^{2}+2 h z\right)}}{1-z^{2}} \int_{z}^{1} e^{\sigma^{\sigma\left(z^{\prime 2}+2 h z^{\prime}\right)}} \mathrm{d} z^{\prime} \mathrm{d} z\right)^{-1}\right]
\end{aligned}
$$

Equation (35) allows one to calculate $\tau_{L}$ for any barrier height. For $\sigma \gg 1$, estimation of the integrals in Eq. (35) using steepest descents yields Eq. (32).

\section{RESULTS AND DISCUSSION}

The longest relaxation time $\tau_{L}$ yielded by Eq. (17), its analytic approximation $\tau_{M}$ from the turnover formula [Eq. (24)], and the integral relaxation time $\tau / \eta$ [Eq. (15)] are shown in Figs. 2-4 for various values of the anisotropy parameter $\sigma$, the asymmetry parameter $h$, and the friction coefficient $\beta^{\prime}$. In particular, the numerical solutions for $\tau_{L}$ via matrix continued fractions using Eq. (17) and the turnover formula Eq. (24) for the longest relaxation time $\tau_{L}$ represented as $\tau_{M}$ are compared in Fig. 2. Clearly the turnover formula for the longest relaxation time, bridging the VLD and IHD escape rates as a function of the dissipation parameter $\beta^{\prime}$, yields satisfactory agreement with the numerical results for rodlike molecules in a 3D asymmetric double well potential for all values of $\beta^{\prime}$ and high 


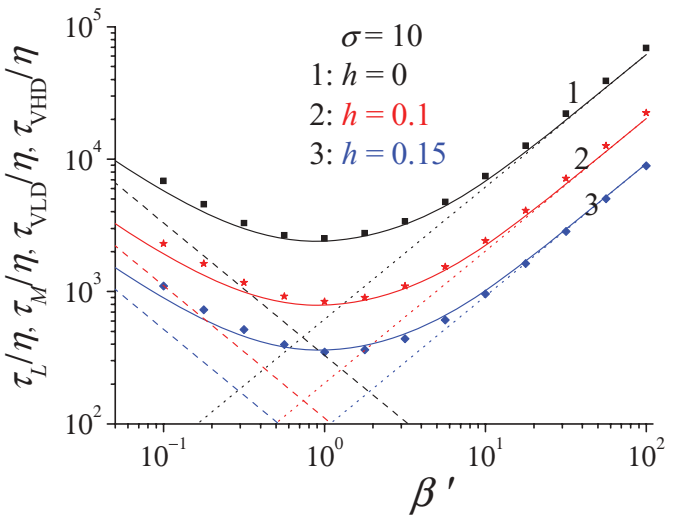

FIG. 2. Characteristic times vs the damping parameter $\beta^{\prime}$ for the barrier height parameter $\sigma=10$ and field parameter $h=0,0.1$, and 0.15 . Symbols: matrix continued fraction solution for the correlation time $\tau$, Eq. (A2). Solid lines: Eq. (30). Dashed and dotted lines: the VLD and VHD asymptotes, Eqs. (31) and (32).

values of $\sigma$. Figures 3 and 4 highlight the effect of the potential asymmetry on the characteristic times. For small values of bias field parameter $h<0.1$, both the correlation time $\tau$ and the longest relaxation time $\tau_{L}$ have very similar Arrhenius behavior (exponential increase with increasing barrier height) so that both may be estimated from the turnover formula. However, for a certain value of $h, h_{c}$ say, the Arrhenius behavior of the correlation time $\tau$ disappears while $\tau_{L}$ still has Arrhenius behavior. This effect occurs at a critical value $h_{c} \approx 0.17$ in this case (the critical field ratio varies from potential to potential) of the ratio $h$, i.e., bias field parameter/anisotropy barrier height parameter, far less than the nucleation field $h_{n}=1$ needed to destroy the bistable nature of the potential. The phenomenon of divergence of the longest relaxation time from the correlation time that appears to be a characteristic feature of certain correlation functions describing relaxation processes in asymmetric bistable potentials ${ }^{50}$ was first discovered from numerical solution of the appropriate hierarchy of the differential recurrence equations by Coffey et al. ${ }^{51}$ for the very similar problem of the longitudinal magnetization relaxation of a uniaxial superparamagnetic particle subjected to a dc magnetic field. The effect was later explained analytically

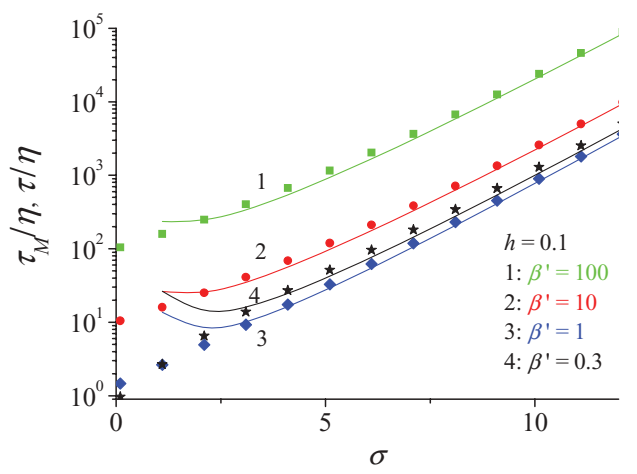

FIG. 3. $\tau$ and $\tau_{M}$ vs the barrier height parameter $\sigma$ for the field parameter $h=0.1$ and various values of the damping parameter $\beta^{\prime}=0.3,1,10$, and 100. Symbols: matrix continued fraction solution for $\tau$, Eq. (A2). Solid lines: Eq. (30).

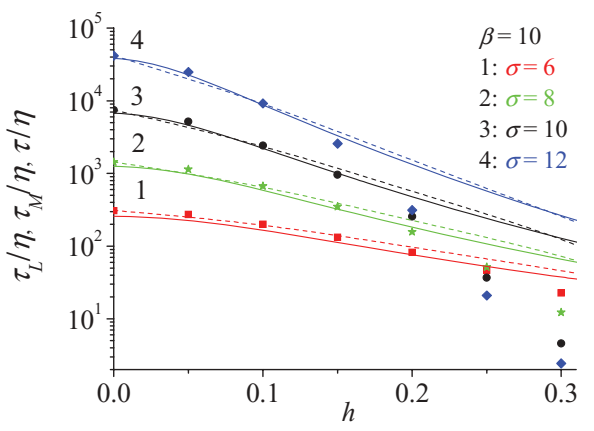

FIG. 4. Characteristic times vs the field parameter $h$ for damping parameter $\beta^{\prime}=10$ and for barrier height parameters $\sigma=6,8,10$, and 12. Symbols: matrix continued fraction solution for $\tau$, Eq. (A2). Solid lines: Eq. (30). Dashed lines: $\tau_{L}$ from Eq. (17).

by Garanin $^{52}$ who showed that it is a natural consequence of the depletion of the population of the shallower of the two potential wells (which are involved in the barrier crossing) by the uniform bias field so that the longitudinal relaxation function becomes insensitive to the exponentially small population of the shallow well. Essentially in spite of the much higher population of the deeper well, escape from this well is a much rarer event than that from the shallow one because of the very high potential barrier which now must be overcome ensuring exponential divergence of the correlation and overbarrier relaxation times.

The real and imaginary parts of the spectrum of the dipole correlation function $\tilde{C}(\omega)$ for various values of the anisotropy parameter $\sigma$ and the asymmetry parameter $h$ are shown in Figs. 5 and 6. In general, three distinct absorption bands appear in the spectrum $\tilde{C}(\omega)$. One absorption band dominates
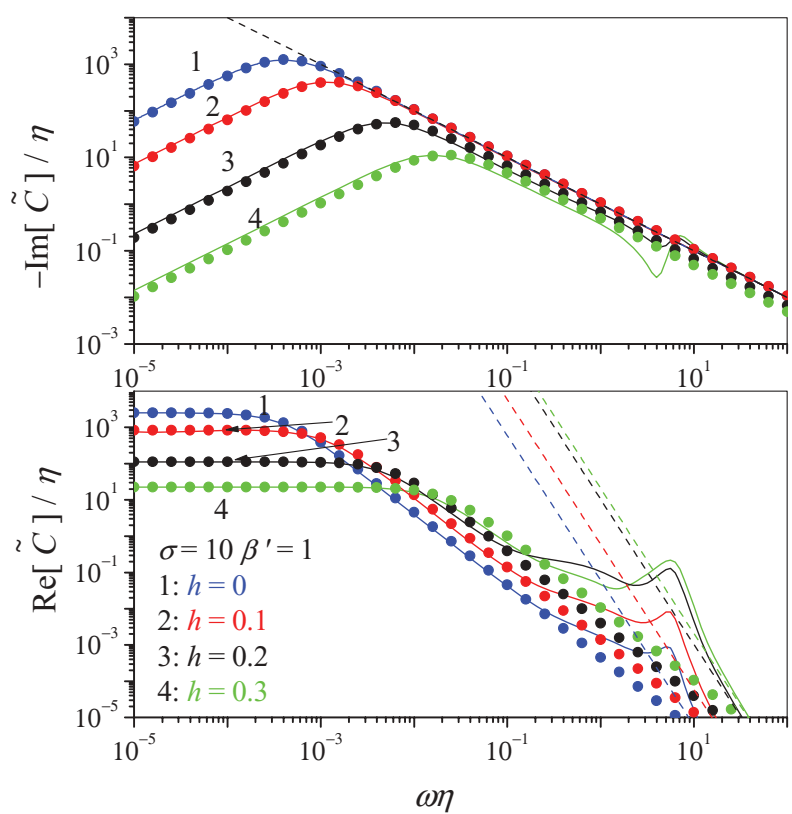

FIG. 5. The real and imaginary parts of the spectra of the dipole correlation function $\tilde{C}(\omega)$ for the friction coefficient $\beta^{\prime}=1$, the barrier parameter $\sigma=$ 10, and various values of the field parameter $h=0,0.1,0.2$, and 0.3. Solid lines: matrix continued fraction solution, Eq. (A2); filled circles: the single Lorentzian Eq. (18); straight dashed lines: the high frequency asymptote Eq. (20). 
the low-frequency part of the spectra and is due to the slow overbarrier Arrhenius relaxation (Kramers transitions) of the dipoles in the $3 \mathrm{D}$ double-well potential. The characteristic frequency $\omega_{R} \approx \lambda_{1}$ of this low-frequency band strongly depends on $\sigma$ and $h$ as well as on the friction parameter $\beta^{\prime}$. Regarding the barrier height or $\sigma$ dependence, the frequency $\omega_{R}$ exponentially decreases as $\sigma$ is raised. This behavior occurs because the probability of escape of a dipole from one well to another over the potential barrier exponentially decreases with increasing $\sigma$ in accordance with the Kramers theory. Clearly from Figs. 5 and 6, the low-frequency part of the spectrum may be approximated by a single Lorentzian Eq. (18). The halfwidths of the spectra $\operatorname{Re} \tilde{C}(\omega)$ shown in Figs. 5 and 6 are determined by $\lambda_{1}^{-1}$ explicitly demonstrating that the overbarrier relaxation mode always partakes in the relaxation process. The second Debye absorption band is due to near degenerate overdamped decay modes in the wells of the potential and lies at intermediate frequencies, i.e., between the low-frequency absorption band due to the overbarrier relaxation and the sharp resonance absorption band at high frequencies. The origin of the latter is the librational motion of dipoles in the wells of the potential due to inertial effects. The high-frequency asymptote Eq. (20) is also shown in Figs. 5 and 6 for comparison.

To conclude we have demonstrated how the matrix continued fraction solution of the hierarchy of recurrence equations generated by the nonlinear Langevin Eqs. (4) and (5) may be successfully applied to the rotational Brownian motion of a linear molecule in space in an asymmetric doublewell potential, Eq. (2), for wide ranges of the barrier height

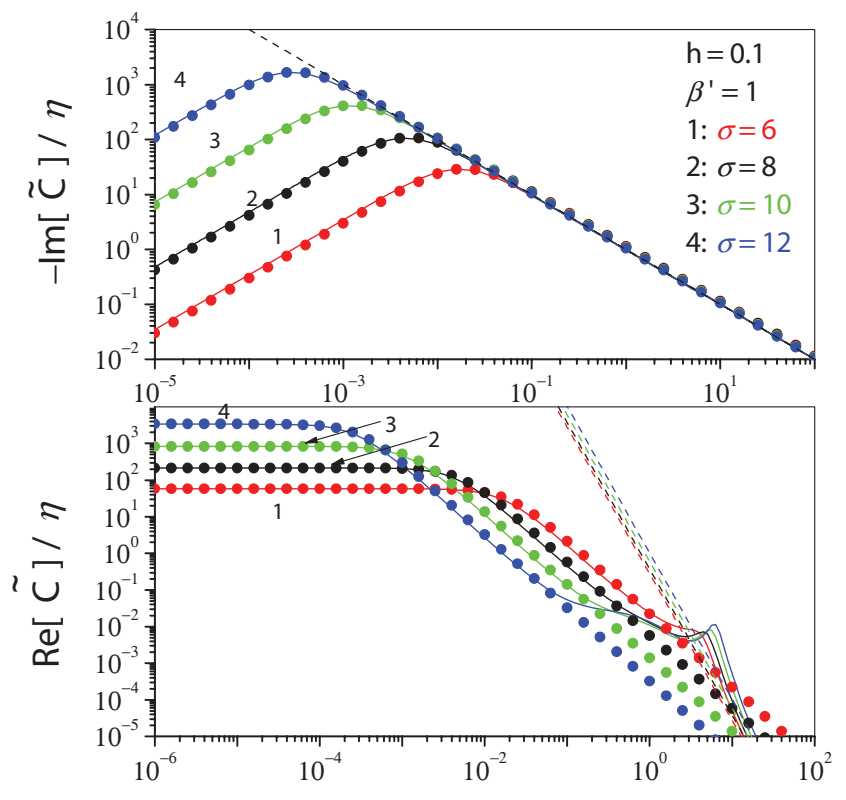

$\omega \eta$

FIG. 6. The real and imaginary parts of the spectra of the dipole correlation function $\tilde{C}(\omega)$ for the friction coefficient $\beta^{\prime}=1$, the field parameter $h=$ 0.1 and various values of the barrier parameter $\sigma=6,8,10$, and 12. Solid lines: matrix continued fraction solution, Eq. (A2); filled circles: the single Lorentzian Eq. (18); straight dashed lines: the high-frequency asymptote Eq. (20). parameter $\sigma$ and the damping parameter $\beta^{\prime}$ (these microscopic parameters are closely associated with the molecular dynamics). We have assumed that every molecule interacts with a mean field potential $V$. Although the mean field approximation has a restricted area of applicability, as it ignores local order effects, the model is easily visualized and allows us to carry out quantitative evaluations of the observables. In particular, we have given simple approximate analytic formulas, Eqs. (24) and (18), for the longest relaxation time namely $\tau_{L} \approx \tau_{M}$ and the low-frequency part of the spec$\operatorname{tra} \tilde{C}(\omega)$ of the dipole autocorrelation function $C(t)$. We have shown that in the low-temperature limit, the turnover formula for the longest relaxation time yields satisfactory agreement with the numerical results for all values of damping. Moreover, it allows one to accurately estimate the damping dependence of the low-frequency part of $\tilde{C}(\omega)$. At low frequencies the model describes the relaxation (Debye) spectrum just as the noninertial rotational diffusion model while in the highfrequency (far-infrared) range the model reproduces the librational (Poley) absorption band and accounts for the excess absorption; i.e., the excess of the intensity of this band over the Debye plateau. ${ }^{43}$ We emphasize that the noninertial rotational diffusion model in a potential is only applicable to the low-frequency range $(\omega \tau<1)$, however, the present model of the inertial rotational diffusion in space in an asymmetric double-well potential may also be used at high frequencies. The results we have given may be easily extended to the so-called itinerant oscillator or cage mode ${ }^{53}$ of rotational motion of molecule in a fluid embodying the suggestion that a typical molecule of a fluid is capable of vibrations about a temporary equilibrium position (cage) which itself undergoes rotational Brownian motion. Hence an additional absorption mechanism due to the slow rotational motion of the cage (similar to the usual Debye free rotational Brownian motion model) will come into play. Thus four absorption mechanisms can be simultaneously explained physically using the same inertia corrected Debye model in the presence of a mean field potential.

\section{APPENDIX: MATRIX CONTINUED FRACTION SOLUTION OF EQS. (13)-(14)}

The hierarchy of differential recurrence Eqs. (13)-(14) for the Fourier coefficients $c_{n}^{l, m}(t)$ can be rearranged as the matrix three-term differential-recurrence equation

$\eta \frac{d}{d t} \mathbf{C}_{n}(t)=\mathbf{Q}_{n}^{-} \mathbf{C}_{n-1}(t)+\mathbf{Q}_{n} \mathbf{C}_{n}(t)+\mathbf{Q}_{n}^{+} \mathbf{C}_{n+1}(t)$.

This is accomplished introducing the vectors

$\mathbf{C}_{n}(t)=\left(\begin{array}{c}\mathbf{c}_{n-1}^{1}(t) \\ \mathbf{c}_{n-1}^{2}(t) \\ \vdots \\ \mathbf{c}_{n-1}^{l_{\max }(t)}\end{array}\right)_{l_{\max }\left(l_{\max }+3\right) / 2}, \ldots \mathbf{c}_{n}^{l}=\left(\begin{array}{c}c_{n}^{l, 0}(t) \\ c_{n}^{l, 1}(t) \\ \vdots \\ c_{n}^{l, l}(t)\end{array}\right)_{l+1}$ 
with $\mathbf{C}_{0}=\mathbf{0}$ and the matrices

$\mathbf{Q}_{n}=\left(\begin{array}{ccccc}\mathbf{q}_{n-1,1} & \mathbf{p}_{n-1,1}^{+} & \mathbf{u}_{n-1,1}^{+} & \ddots & \mathbf{0} \\ \mathbf{p}_{n-1,2}^{-} & \mathbf{q}_{n-1,2} & \mathbf{p}_{n-1,2}^{+} & \ddots & \ddots \\ \mathbf{u}_{n-1,3}^{-} & \mathbf{p}_{n-1,3}^{-} & \ddots & \ddots & \mathbf{u}_{n-1, l_{\max }-2}^{-} \\ \ddots & \ddots & \ddots & \ddots & \mathbf{p}_{n-1, l_{\max }-1}^{+} \\ \mathbf{0} & \ddots & \mathbf{u}_{n-1, l_{\max }}^{-} & \mathbf{p}_{n-1, l_{\max }}^{-} & \mathbf{q}_{n-1, l_{\max }}\end{array}\right)$

$\mathbf{Q}_{n}^{-}=\left(\begin{array}{ccccc}\mathbf{q}_{n-1,1}^{-} & \mathbf{r}_{n-1,1}^{+} & \mathbf{v}_{n-1,1}^{+} & \ddots & \mathbf{0} \\ \mathbf{r}_{n-1,2}^{-} & \mathbf{q}_{n-1,2}^{-} & \mathbf{r}_{n-1,2}^{+} & \ddots & \ddots \\ \mathbf{v}_{n-1,3}^{-} & \mathbf{r}_{n-1,3}^{-} & \ddots & \ddots & \mathbf{v}_{n-1, l_{\max }-2}^{+} \\ \ddots & \ddots & \ddots & \mathbf{q}_{n-1, l_{\max }-1}^{-} & \mathbf{r}_{n-1, l_{\max }-1}^{+} \\ \mathbf{0} & \ddots & \mathbf{v}_{n-1, l_{\max }}^{-} & \mathbf{r}_{n-1, l_{\max }}^{-} & \mathbf{q}_{n-1, l_{\max }}^{-}\end{array}\right)$

$\mathbf{Q}_{n}^{+}=\left(\begin{array}{ccccc}\mathbf{q}_{n-1,1}^{+} & \mathbf{0} & \mathbf{0} & \cdots & \mathbf{0} \\ \mathbf{0} & \mathbf{q}_{n-1,2}^{+} & \mathbf{0} & \ldots & \mathbf{0} \\ \mathbf{0} & \mathbf{0} & \ddots & \ddots & \vdots \\ \vdots & \vdots & \ddots & \mathbf{q}_{n-1, l_{\max }-1}^{+} & \mathbf{0} \\ \mathbf{0} & \mathbf{0} & \cdots & \mathbf{0} & \mathbf{q}_{n-1, l_{\max }^{+}}^{+}\end{array}\right)$,

where $\mathbf{q}_{n, l}, \mathbf{q}_{n, l}^{ \pm}, \mathbf{r}_{n, l}^{ \pm}, \mathbf{p}_{n, l}^{ \pm} \mathbf{u}_{n, l}^{ \pm}$, and $\mathbf{v}_{n, l}^{ \pm}$are

$\underset{\left[\mathbf{q}_{n, l}\right]_{i j}}{i, j=1,2, \ldots l+1}=\left\{\begin{array}{l}\frac{1}{4}\left(1+\delta_{i, 1}\right) \delta_{i, j-1}-2 n \beta^{\prime} \delta_{i, j}-\frac{1}{4}\left(n-\frac{i-3}{2}\right)(l+i-1)(l-i+2) \delta_{i, j+1}, \quad i: \text { odd } \\ {\left[1+\frac{2 \sigma(2 i-1)}{(2 l-1)(2 l+3)}\right] \delta_{i, j-1}-(2 n+1) \beta^{\prime} \delta_{i, j}} \\ -\left(n+\frac{i}{2}\right)(l+i-1)(l-i+2)\left[1-\frac{2 \sigma(2 i-3)}{(2 l-1)(2 l+3)}\right] \delta_{i, j+1}, \quad i: \text { even }\end{array}\right.$

$\left.\underset{i, j=1,2, \ldots l+1}{\left[\mathbf{q}_{n, l}^{+}\right.}\right]_{i j}=\frac{1}{4}\left[\delta_{i, j-1}-\left(n-\frac{i}{2}+2\right)(l+i-1)(l-i+2) \delta_{i, j+1}\right], \quad i:$ even

$\underset{i, j=1,2, \ldots l+1}{\left[\mathbf{q}_{n, l}^{-}\right]_{i j}}=\left[1+\frac{2 \sigma(2 i-1)}{(2 l-1)(2 l+3)}\right]\left(1+\delta_{i, 1}\right) \delta_{i, j-1}$

$-\left(n+\frac{i-1}{2}\right)(l+i-1)(l-i+2)\left[1-\frac{2 \sigma(2 i-3)}{(2 l-1)(2 l+3)}\right] \delta_{i, j+1}, \quad i:$ odd

$\underset{\substack{i=1,2, \ldots l+1 \\ j=1,2, \ldots l+2}}{\left[\mathbf{p}_{n, l}^{+}\right]_{i j}}=\frac{\xi}{2 l+1}\left[\delta_{i, j-1}-\left(n+\frac{i}{2}\right)(l-i+2)(l-i+3) \delta_{i, j+1}\right], \quad i:$ even

$\underset{\substack{i=1,2, \ldots l+1 \\ j=1,2, l}}{\left[\mathbf{p}_{n, l}^{-}\right]_{i j}}=-\frac{\xi}{2 l+1}\left[\delta_{i, j-1}-\delta_{i, j+1}\left(n+\frac{i}{2}\right)(l+i-2)(l+i-1)\right], \quad i:$ even $\underset{\substack{i=1,2, \ldots l+1 \\ j=1,2, \ldots l+2}}{\left[\mathbf{r}_{n, 1}^{+}\right]_{i j}}=\frac{\xi}{2 l+1}\left[\left(1+\delta_{i, 1}\right) \delta_{i, j-1}-\left(n+\frac{i-1}{2}\right)(l-i+3)(l-i+2) \delta_{i, j+1}\right], \quad i:$ odd

$\underset{\substack{i=1,2, \ldots l+1 \\\left[\mathbf{r}_{n,-}^{-}\right]_{i j}}}{ }=-\frac{\xi}{2 l+1}\left[\left(1+\delta_{i, 1}\right) \delta_{i, j-1}-\left(n+\frac{i-1}{2}\right)(l+i-1)(l+i-2) \delta_{i, j+1}\right], \quad i:$ odd $i=1,2, \ldots l+1$
$j=1,2, \ldots l$

$\underset{\substack{i=1,2, \ldots l+1 \\ j=1,2, \ldots l+3}}{\left[\mathbf{u}_{n, l}^{+}\right]_{i j}}=\frac{2 \sigma(l-i+2)}{(2 l+1)(2 l+3)}\left[\delta_{i, j-1}-\left(n+\frac{i}{2}\right)(l-i+3)(l-i+4) \delta_{i, j+1}\right], \quad i$ : even $\underset{\substack{i=1,2, \ldots l+1 \\ j=1,2, \ldots l-1}}{\left[\mathbf{u}_{n, l}^{-}\right]_{i j}}=-\frac{2 \sigma(l+i-1)}{(2 l-1)(2 l+1)}\left[\delta_{i, j-1}-\left(n+\frac{i}{2}\right)(l+i-2)(l+i-3) \delta_{i, j+1}\right], \quad i:$ even 


$$
\begin{aligned}
& \underset{\substack{i=1,2, \ldots l+1 \\
j=1,2, \ldots l+3}}{\left[\mathbf{v}_{n, l}^{+}\right]_{i j}}=\frac{2 \sigma(l-i+2)}{(2 l+1)(2 l+3)}\left[\left(1+\delta_{i, 1}\right) \delta_{i, j-1}-\left(n+\frac{i-1}{2}\right)(l-i+3)(l-i+4) \delta_{i, j+1}\right], \quad i: \text { odd } \\
& \underset{\substack{i=1,2, \ldots l+1 \\
j=1,2, \ldots l-1}}{\left[\mathbf{v}_{n, l}^{-}\right]_{i j}}=-\frac{2 \sigma(l+i-1)}{(2 l-1)(2 l+1)}\left[\left(1+\delta_{i, 1}\right) \delta_{i, j-1}-\left(n+\frac{i-1}{2}\right)(l+i-2)(l+i-3) \delta_{i, j+1}\right], \quad i: \text { odd. }
\end{aligned}
$$

All other elements in these matrixes are zero.

The exact solution of Eq. (A1) for the Laplace transform $\tilde{\mathbf{C}}_{1}(s)=\int_{0}^{\infty} \mathbf{C}_{1}(t) e^{-s t} \mathrm{~d} t$ is then rendered in terms of matrix continued fractions as ${ }^{2}$

$$
\tilde{\mathbf{C}}_{1}(s)=\eta \Delta_{1}(s) \mathbf{C}_{1}(0),
$$

where

$$
\begin{aligned}
& \Delta_{n}(s) \\
& =\frac{\mathbf{I}_{n}}{\eta s \mathbf{I}_{n}-\mathbf{Q}_{n}-\mathbf{Q}_{n}^{+} \frac{\mathbf{I}_{n+1}}{\eta s \mathbf{I}_{n+1}-\mathbf{Q}_{n+1}-\mathbf{Q}_{n+1}^{+} \frac{\mathbf{I}_{n+2}}{{ }_{n s \mathbf{I}_{n+2}-\mathbf{Q}_{n+2}} \ddots} \mathbf{Q}_{n+2}^{-}} \mathbf{Q}_{n+1}^{-}} .
\end{aligned}
$$

The initial value vector $\mathbf{C}_{1}(0)$ in Eq. (A3) is given by

$$
\mathbf{C}_{1}(0)=\left(\begin{array}{c}
\mathbf{c}_{0}^{1}(0) \\
\mathbf{c}_{0}^{2}(0) \\
\vdots \\
\mathbf{c}_{0}^{l_{\max }(0)}
\end{array}\right)_{l_{\max }\left(l_{\max }+3\right) / 2}, \ldots \mathbf{c}_{0}^{l}(0)=\left(\begin{array}{c}
c_{0}^{l, 0} \\
0 \\
\vdots \\
0
\end{array}\right)_{l+1}
$$

where

$$
\begin{aligned}
c_{0}^{l, 0} & =\left\langle P_{1} P_{l}\right\rangle-\left\langle P_{1}\right\rangle\left\langle P_{l}\right\rangle \\
& =\frac{l+1}{2 l+1}\left\langle P_{l+1}\right\rangle+\frac{l}{2 l+1}\left\langle P_{l-1}\right\rangle-\left\langle P_{1}\right\rangle\left\langle P_{l}\right\rangle .
\end{aligned}
$$

Here $c_{n}^{l, m}(0)=0$, for $n \neq 0$ and $m \neq 0$. The functions $\left\langle P_{l}\right\rangle$ satisfy the recurrence equation ${ }^{2}$

$$
\begin{aligned}
& \left(1-\frac{2 \sigma}{(2 l-1)(2 l+3)}\right)\left\langle P_{l}\right\rangle+\frac{\xi}{2 l+1}\left(\left\langle P_{l+1}\right\rangle-\left\langle P_{l-1}\right\rangle\right) \\
& +\frac{2 \sigma(l+2)}{(2 l+1)(2 l+3)}\left\langle P_{l+2}\right\rangle-\frac{2 \sigma(l-1)}{(2 l-1)(2 l+1)}\left\langle P_{l-2}\right\rangle=0,
\end{aligned}
$$

and can be found $a^{2}\left(\left\langle P_{0}\right\rangle=1\right)$,

$$
\left(\begin{array}{c}
\left\langle P_{2 l}\right\rangle \\
\left\langle P_{2 l-1}\right\rangle
\end{array}\right)=\mathbf{S}_{l} \cdots \mathbf{S}_{2} \mathbf{S}_{1}\left(\begin{array}{c}
\left\langle P_{0}\right\rangle \\
0
\end{array}\right)
$$

where the matrix continued fraction $\mathbf{S}_{l}$ is defined by the recurrence equation

$$
\mathbf{S}_{l}=\left[\mathbf{z}_{l}+\mathbf{z}_{l}^{+} \mathbf{S}_{l+1}\right]^{-1} \mathbf{z}_{l}^{-},
$$

with

$$
\mathbf{z}_{l}=\left(\begin{array}{cc}
1-\frac{2 \sigma}{(4 l-1)(4 l+3)} & -\frac{\xi}{4 l+1} \\
\frac{\xi}{4 l-1} & 1-\frac{2 \sigma}{(4 l-3)(4 l+1)}
\end{array}\right),
$$

$$
\begin{aligned}
& \mathbf{z}_{l}^{-}=\left(\begin{array}{cc}
\frac{2 \sigma(2 l-1)}{16 l^{2}-1} & 0 \\
\frac{\xi}{4 l-1} & \frac{2 \sigma(2 l-2)}{(4 l-3)(4 l-1)}
\end{array}\right), \\
& \mathbf{z}_{l}^{+}=\left(\begin{array}{cc}
\frac{2 \sigma(2 l+2)}{(4 l+1)(4 l+3)} & \frac{\xi}{4 l+1} \\
0 & \frac{2 \sigma(2 l+1)}{16 l^{2}-1}
\end{array}\right) .
\end{aligned}
$$

We remark that the initial value $\dddot{C}(0)=\dddot{c}_{0}^{1,0}(0) / c_{0}^{1,0}(0)$ can be determined from the vectors $\dddot{\mathbf{C}}_{1}(0)$ and $\mathbf{C}_{1}(0)$. Noting that $\mathbf{C}_{n}(0)=\mathbf{0}$ for $n>1$, one can show that the third derivative of the vector $\mathbf{C}_{1}(t)$ at $t=0$ is

$$
\begin{aligned}
\eta^{3} \dddot{\mathbf{C}}_{1}(0)= & {\left[\mathbf{Q}_{1}\left(\mathbf{Q}_{1} \mathbf{Q}_{1}+\mathbf{Q}_{1}^{+} \mathbf{Q}_{2}^{-}\right)\right.} \\
& \left.+\mathbf{Q}_{1}^{+}\left(\mathbf{Q}_{2}^{-} \mathbf{Q}_{1}+\mathbf{Q}_{2} \mathbf{Q}_{2}^{-}\right)\right] \mathbf{C}_{1}(0) .
\end{aligned}
$$

The smallest nonvanishing eigenvalue $\lambda_{1}$ of the FokkerPlanck operator can also be estimated by using matrix continued fractions from the secular equation as ${ }^{1,2}$

$$
\operatorname{det}\left[\lambda_{1} \tau_{N} \mathbf{I}+\mathbf{Q}_{1}+\mathbf{Q}_{1}^{+} \Delta_{2}\left(-\lambda_{1}\right) \mathbf{Q}_{2}^{-}\right]=0 .
$$

The matrix continued fraction solution Eq. (A3) we have obtained is easily computed (algorithms for calculating matrix continued fractions are discussed in Refs. 1 and 2). As far as practical calculations of matrix continued fractions to the present problem are concerned, we approximate it by matrix continued fractions of finite order (by setting during the calculations the value of $l_{\max }$ in Eqs. (A2) and (A4) in such a way that a further increase of $l_{\max }$ did not change the result $\left(l_{\max }\right.$ depends on the dimensionless barrier $\sigma$ and damping $\beta^{\prime}$ parameters and must be chosen taking into account the desired degree of accuracy of the calculation). With increasing $\sigma$ and decreasing $\beta, l_{\max }$ increases. For very low damping, $\beta^{\prime}<0.1$, however, the method is not applicable because the matrixes involved become ill conditioned so that numerical inversions are no longer possible.

${ }^{1}$ H. Risken, The Fokker-Planck Equation, 2nd ed. (Springer-Verlag, Berlin, 1989).

${ }^{2}$ W. T. Coffey, Yu. P. Kalmykov, and J. T. Waldron, The Langevin Equation, 2nd ed. (World Scientific, Singapore, 2004). 
${ }^{3}$ E. Hershkovits and R. Hernandez, J. Chem. Phys. 122, 014509 (2005).

${ }^{4}$ H. A. Kramers, Physica (Utrecht) 7, 284 (1940).

${ }^{5}$ P. Hänggi, P. Talkner, and M. Borkovec, Rev. Mod. Phys. 62, 251 (1990).

${ }^{6}$ W. T. Coffey, Adv. Chem. Phys. 103, 259 (1998).

${ }^{7}$ E. Pollak and P. Talkner, Chaos 15, 026116 (2005).

${ }^{8}$ P. Hänggi, J. Stat. Phys. 42, 105 (1986); 44, 1003 (1986).

${ }^{9}$ W. T. Coffey, D. A. Garanin, and D. J. McCarthy, Adv. Chem. Phys. 117, 483 (2001).

${ }^{10}$ V. I. Mel'nikov, Physica A 130, 606 (1985).

${ }^{11}$ V. I. Mel'nikov and S. V. Meshkov, J. Chem. Phys. 85, 1018 (1986).

${ }^{12}$ V. I. Mel'nikov, Phys. Rep. 209, 1 (1991).

${ }^{13}$ H. Grabert, Phys. Rev. Lett. 61, 1683 (1988).

${ }^{14}$ E. Pollak, H. Grabert, and P. Hänggi, J. Chem. Phys. 91, 4073 (1989).

${ }^{15}$ M. Topaler and N. Makri, J. Chem. Phys. 101, 7500 (1994).

${ }^{16}$ A. N. Drozdov and P. Talkner, J. Chem. Phys. 109, 2080 (1998).

${ }^{17}$ H. X. Zhou, Chem. Phys. Lett. 164, 285 (1989).

${ }^{18}$ R. Ferrando, R. Spadacini, and G. E. Tommei, Phys. Rev. A 46, R699 (1992); Phys. Rev. E 48, 2437 (1993); R. Ferrando, R. Spadacini, G. E. Tommei, and V. I. Mel'nikov, ibid. 51, R1645 (1995).

${ }^{19}$ Yu. P. Kalmykov, W. T. Coffey, and S. V. Titov, J. Chem. Phys. 124, 024107 (2006); W. T. Coffey, Yu. P. Kalmykov, and S. V. Titov, Phys. Rev. E 73, 061101 (2006).

${ }^{20}$ E. Pollak, J. Bader, B. J. Berne, and P. Talkner, Phys. Rev. Lett. 70, 3299 (1993).

${ }^{21}$ Yu. Georgievskii and E. Pollak, Phys. Rev. E 49, 5098 (1994).

${ }^{22}$ E. Hershkovits, P. Talkner, E. Pollak, and Yu. Georgievskii, Surf. Sci. 421, 73 (1999).

${ }^{23}$ R. W. Pastor and A. Szabo, J. Chem. Phys. 97, 5098 (1992).

${ }^{24}$ W. T. Coffey, Yu. P. Kalmykov, and S. V. Titov, J. Chem. Phys. 120, 9199 (2004).

${ }^{25}$ Yu. P. Kalmykov, S. V. Titov, and W. T. Coffey, J. Chem. Phys. 123, 094503 (2005).

${ }^{26}$ Yu. P. Kalmykov, S. V. Titov, and W. T. Coffey, J. Chem. Phys. 130, 064110 (2009)

${ }^{27}$ P. M. Déjardin, D. S. F. Crothers, W. T. Coffey, and D. J. McCarthy, Phys. Rev. E 63, 021102 (2001); Yu. P. Kalmykov, J. Appl. Phys. 96, 1138 (2004); Yu. P. Kalmykov, W. T. Coffey, B. Ouari, and S. V. Titov, J. Magn. Magn. Mater. 292, 372 (2005); Yu. P. Kalmykov, W. T. Coffey, and S. V. Titov, Phys. Solid. State 47, 272 (2005); W. T. Coffey, P. M. Déjardin, and Yu. P. Kalmykov, Phys. Rev. B 79, 054401 (2009); B. Ouari, S. Aktaou, and Yu. P. Kalmykov, Phys. Rev. B 81, 024412 (2010).

${ }^{28}$ S. I. Denisov, K. Sakmann, P. Talkner, and P. Hänggi, Europhys. Lett. 76, 1001 (2006); S. I. Denisov, T. V. Lyutyy, and P. Hänggi, Phys. Rev. Lett. 97, 227202 (2006)

${ }^{29}$ G. Meier and A. Saupe, Mol. Cryst. 1, 515 (1966).
${ }^{30}$ A. J. Martin, G. Meier, and A. Saupe, Symp. Faraday Soc. 5, 119 (1971).

${ }^{31}$ P. L. Nordio and P. Busolin, J. Chem. Phys. 55, 5485 (1971); P. L. Nordio, G. Rigatti, and U. Segre, ibid. 56, 2117 (1971); Mol. Phys. 25, 129 (1973).

${ }^{32}$ B. A. Storonkin, Kristallogr. 30, 841 (1985) [Sov. Phys. Crystallogr. 30, 489 (1985)]; F. M. Kuni, A. A. Melikhov, and B. A. Storonkin, Theor. Math. Phys. 34, 374 (1978).

${ }^{33}$ Yu. P. Kalmykov and W. T. Coffey, Liquid Crystals 25, 329 (1998); W. T. Coffey and Yu. P. Kalmykov, Adv. Chem. Phys. 113, 487 (2000).

${ }^{34}$ B. U. Felderhof, Physica A 323, 88 (2003).

${ }^{35}$ M. L. Huertas, V. Cruz, J. J. Lopez Cascales, A. U. Acuha, and J. Garcia de la Torre, Biophys. J. 71, 1428 (1996).

${ }^{36}$ M. X. Fernandes, M. L. Huertas, M. A. R. B. Castanho, and J. García de la Torre, Biophys. Chem. 79, 41 (1999).

${ }^{37}$ B. Lassier and C. Brot, Discuss. Faraday Soc. 48, 39 (1969); C. Brot and I. Darmont, Mol. Phys. 21, 785 (1971); R. W. Gerling and B. de Raedt, J. Chem. Phys. 77, 6263 (1982).

${ }^{38}$ C. J. Reid, Mol. Phys. 49, 331 (1983); F. Marchesoni and J. K. Vij, Z. Phys. B: Condens. Matter 58, 187 (1985); F. Marchesoni, Phys. Rev. B 32, 1827 (1985); W. T. Coffey, C. Rybarsch, and W. Schröer, Phys. Letts. A 88, 331 (1982)

${ }^{39}$ A. I. Burshtein and S. I. Temkin, Spectroscopy of Molecular Rotation in Gases and Liquids (Cambridge University Press, Cambridge, 1994).

${ }^{40}$ J. R. McConnell, Rotational Brownian Motion and Dielectric Theory (Academic, New York, 1980).

${ }^{41}$ W. T. Coffey, J. Chem. Phys. 93, 724 (1990); ibid. 95, 2026 (1991); ibid. 99, 3014 (1993).

${ }^{42}$ S. V. Titov, Yu. P. Kalmykov, and W. T. Coffey, J. Chem. Phys. 129, 144505 (2008).

${ }^{43}$ M. W. Evans, G. J. Evans, W. T. Coffey, and P. Grigolini, Molecular Dynamics and Theory of Broadband Spectroscopy (Wiley, New York, 1982).

${ }^{44}$ Handbook of Mathematical Functions, edited by M. Abramowitz and I. Stegun (Dover, New York, 1964).

${ }^{45}$ W. T. Coffey, Yu. P. Kalmykov, and S. V. Titov, J. Phys. A: Math. Theor. 35, 6789 (2002).

${ }^{46}$ R. A. Sack, Proc. Phys. Soc. B 70, 402 (1957); ibid. 70, 414 (1957).

${ }^{47}$ P. Jung and P. Hänggi, Phys. Rev. Lett. 61, 11 (1988).

${ }^{48} \mathrm{H}$. Goldstein, Classical Mechanics, 2nd ed. (Addison-Wesley, Reading, MA, 1980).

${ }^{49}$ P. Debye, Polar Molecules (Chemical Catalog Co., New York, 1929); reprinted by Dover, New York, 1954.

${ }^{50}$ W. T. Coffey, D. S. F. Crothers, and Yu. P. Kalmykov, Phys. Rev. E 55, 4812 (1997).

${ }^{51}$ W. T. Coffey, D. S. F. Crothers, Yu. P. Kalmykov, and J. T. Waldron, Phys. Rev. B 51, 15947 (1995).

${ }^{52}$ D. A. Garanin, Phys. Rev. E 54, 3250 (1996).

${ }^{53}$ W. T. Coffey, Yu. P. Kalmykov, and S. V. Titov, Adv. Chem. Phys. 126, 131 (2003). 